\title{
Article
}

\section{Risk of Enteric Infection in Patients with Gastric Acid Supressive Drugs: A Population-Based Case-Control Study}

\author{
Chia-Jung Kuo ${ }^{1,2,3}$, Cheng-Yu Lin ${ }^{1,2}$, Chun-Wei Chen ${ }^{1,2}$, Chiu-Yi Hsu ${ }^{4}{ }^{\mathbb{C}}$, Sen-Yung Hsieh ${ }^{1,2}$, \\ Cheng-Tang Chiu ${ }^{1,2,3}$ and Wey-Ran Lin $1,2,5, *$ (D)
}

1 Department of Gastroenterology and Hepatology, Linkou Chang Gung Memorial Hospital, Taoyuan 333, Taiwan; m7011@cgmh.org.tw (C.-J.K.); 8805035@cgmh.org.tw (C.-Y.L.); 8902088@cgmh.org.tw (C.-W.C.); siming.shia@gmail.com (S.-Y.H.); ctchiu@adm.cgmh.org.tw (C.-T.C.)

2 Department of Gastroenterology, Chang Gung Memorial Hospital at Linkou, College of Medicine, Chang Gung University, Taoyuan 333, Taiwan

3 Chang Gung Microbiota Therapy Center, Linkou Chang Gung Memorial Hospital, Taoyuan 333, Taiwan

4 Center for Big Data Analytics and Statistics, Linkou Chang Gung Memorial Hospital, Taoyuan 333, Taiwan; joy960111@gmail.com

5 Department of Gastroenterology and Hepatology, Chang Gung Memorial Hospital, 5, Fushin Street, Kweishan, Taoyuan 333, Taiwan

* Correspondence: t12360@adm.cgmh.org.tw; Tel.: +886-3-3281200 (ext. 8102); Fax: +886-3-3272236

Citation: Kuo, C.-J.; Lin, C.-Y.; Chen, C.-W.; Hsu, C.-Y.; Hsieh, S.-Y.; Chiu, C.-T.; Lin, W.-R. Risk of Enteric Infection in Patients with Gastric Acid Supressive Drugs: A Population-Based Case-Control Study. J. Pers. Med. 2021, 11, 1063. https:// doi.org/10.3390/jpm11111063

Academic Editor: Jorge Luis Espinoza

Received: 16 July 2021

Accepted: 19 October 2021

Published: 22 October 2021

Publisher's Note: MDPI stays neutral with regard to jurisdictional claims in published maps and institutional affiliations.

Copyright: (C) 2021 by the authors. Licensee MDPI, Basel, Switzerland. This article is an open access article distributed under the terms and conditions of the Creative Commons Attribution (CC BY) license (https:// creativecommons.org/licenses/by/ $4.0 /)$.

\begin{abstract}
Abstract: BackgroundLong-term use of gastric-acid-suppressive drugs is known to be associated with several adverse effects. However, the association between enteric infection and acid suppression therapy is still uncertain. This study aimed to evaluate the association between gastric acid suppression and the risk of enteric infection. Materials and Methods: We conducted a population-based case-control study using the data from Chang Gung Research Database (CGRD) in Taiwan. Between January 2008 and December 2017, a total of 154,590 adult inpatients (age > 18) were identified. A pool of potential eligible controls according to four propensity scores matching by sex, age, and index year were extracted $(n=89,925)$. Subjects with missing data or who received less than 7 days of proton pump inhibitors (PPIs) and/or H2-receptor antagonists (H2RAs) were excluded. Finally, 17,186 cases and 69,708 corresponding controls were selected for analysis. The use of PPIs and H2RAs, the result of microbiological samples, and co-morbidity conditions have been analyzed. Confounders were controlled by conditional logistic regression. Results: $32.84 \%$ of patients in the case group used PPIs, compared with $7.48 \%$ in the control group. Of patients in the case group, $9.9 \%$ used H2RAs, compared with $6.9 \%$ in the control group. Of patients in the case group, $8.3 \%$ used a combination of PPIs and H2RAs, compared with $2.7 \%$ in the control group. The most common etiological pathogens were Enterococcus (44.8\%), Clostridioides difficile (34.5\%), and Salmonella spp. (10.2\%). The adjusted odds ratio (OR) for PPI use with enteric infection was 5.526 (95\% confidence interval [CI], 5.274-5.791). For H2RAs, the adjusted odds ratio was 1.339 (95\% confidence interval [CI], 1.261-1.424). Compared to the control group, persons with enteric infection had more frequent acid-suppressive agent usage. Conclusions: This study demonstrates that gastric-acid-suppressive drug use is associated with an increased risk of enteric infection after adjusting for potential biases and confounders.
\end{abstract}

Keywords: enteric infection; proton pump inhibitor; H2-receptor antagonists

\section{Introduction}

Proton pump inhibitors (PPIs) inhibit the secretion of hydrogen ions into the stomach by inhibiting the $\mathrm{H}+/ \mathrm{K}+$ ATPase enzyme present in gastric parietal cells. The action results in prolonged elevation of intragastric $\mathrm{pH}$ levels and is commonly used to treat gastric-acid-related diseases such as upper gastrointestinal bleeding, peptic ulcers, and gastroesophageal reflux [1-3]. PPIs are considered well tolerated and highly efficacious. 
However, emerging studies have suggested that long-term use of PPIs may be associated with several adverse effects, such as bacterial pneumonia, osteoporotic-related fractures, kidney disease, impaired absorption of nutrients, ischemic stroke, cardiovascular events, and even, the risk of cancer [1,4-12]. Long-term acid suppression facilitates the development of fundic ECL cell hyperplasia, overgrowth of non-Helicobacter pylori bacterial species, lower the acid bactericidal effects for harmful microorganisms and alters the natural course of Helicobacter pylori gastritis, transforming the antral-predominant pattern into a body-predominant pattern [13]. A link between gastric-acid-suppressive drugs and increased enteric infection risk is based on several potential hypotheses. By reducing the secretion of hydrochloric acid produced by the stomach, PPIs and H2RAs may promote the growth of gastrointestinal pathogenic microflora, increase bacterial translocation, affect the gastrointestinal microbiome, PPIs therapy also inhibited the neutrophil's bactericidal activity [14].

Previous investigation in Western countries reported that there is an association between acid suppression drug use and increased risk of enteric infection [15]. Furthermore, the effect was related to the degree of gastric acid inhibition and greater for PPI use compared with H2RA use. There was a trend for the association to be stronger for Salmonella, Campylobacter, or Shigella infection [15]. The association between long -term PPIs use and Clostridioides difficile infection is also reported [16-18].

Metabolism of PPI depends on hepatic cytochrome P450 enzymes, especially the CYP2C19 genotype, and has different activity due to gene polymorphism. Genetic polymorphism of CYP2C19 shows marked interracial differences, with the poor metabolizer phenotype representing $2 \sim 5 \%$ of Caucasian and up to $11 \sim 23 \%$ of Oriental population [19]. Poor metabolism of PPI results in greater bioavailability and subsequently increased antisecretory efficacy.

Our current study aims to evaluate the association between gastric-acid-suppressive drug use and the risk of enteric infection for Asian population.

\section{Materials and Methods}

\subsection{Ethics Statement}

Ethics approval was obtained from the Institutional Review Board of CGMH (No.201900774B0C601). As all data were anonymized from existing databases and results were presented in aggregate, the requirement for informed consent was waived according to IRB regulations.

\subsection{Data Source}

Data were obtained from the Chang Gung Research Database (CGRD). The Chang Gung Medical Foundation (CGMF), which consists of seven Chang Gung Memorial Hospitals (CGMHs), is the largest medical system in Taiwan. CGMF has 10,070 beds and admits more than 280,000 patients per year. All seven CGMHs use electronic medical records (EMRs). The CGRD is an anonymized database comprising of multi-institutional standardized EMRsn [20]. This study is based in part on data from the CGRD provided by CGMHs. The interpretation and conclusions contained herein do not represent the position of CGMHs.

\subsection{Study Population}

We conducted a case control study using data from CGRD in Taiwan. We used the International Classification of Diseases (ICD-9-CM and ICD-10-CM) to define diseases. Case subjects were identified from the CGRD by using inpatient discharge records. Between January 2008 and December 2017, a total of 154,590 adult inpatients (age > 18) were identified. A pool of potential eligible controls with the same follow-up period as the case patient according to 4 propensity scores matching by sex, age, and index year were extracted $(n=89,925)$. Because the therapeutic doses of PPIs or H2Bs reach a steady state after daily dosing and thus achieve their maximal effective level between 5 to $7 \mathrm{~d}$, 
subjects who received less than 7 days of drugs were excluded. Finally, 17,186 cases and 69,708 corresponding controls were selected for analysis. The flow chart illustrates inclusion and exclusion in the current study (Figure 1).

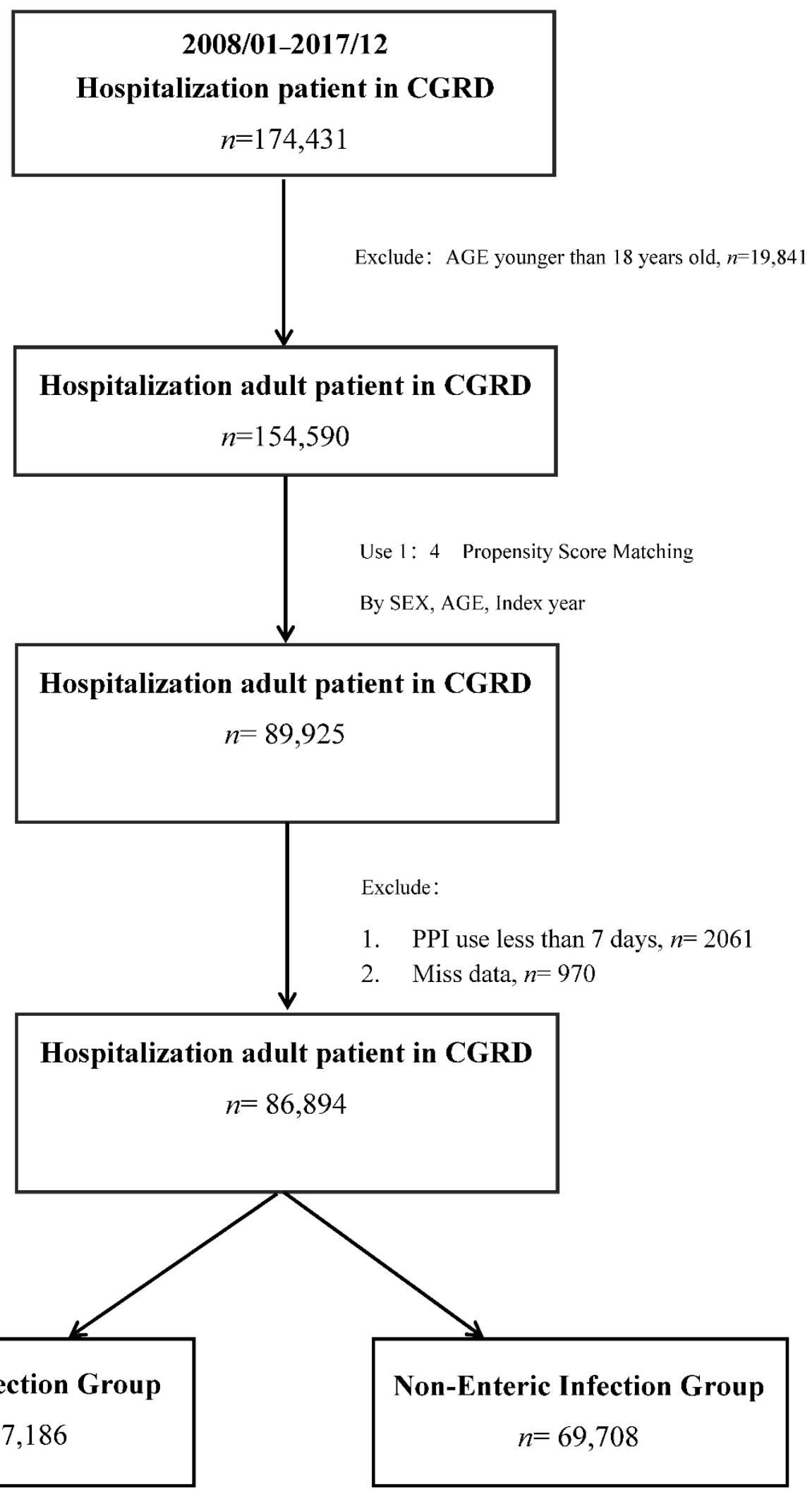

Figure 1. Flow chart of current study. 


\subsection{Exposure Assessment}

Information on the prescribed drugs was extracted from the CGRD. We identified all PPIs and H2RAs prescribed within 6 months before the index date; PPIs included pantoprazole, lansoprazole, rabeprazole, esomeprazole, and dexlansoprazole. H2RAs included famotidine and cimetidine. The defined daily doses (DDDs) recommended by the World Health Organization were used to quantify the use of PPIs or H2RAs. Cumulative DDD was estimated as the sum of dispensed DDD for any PPI or H2RA, and the final dose was defined as the latest dose taken within the specified period prior to the index date. Collected data included date of prescription, daily dosage, and number of days on the drug.

\subsection{Statistical Analysis}

For comparison, the chi-square statistics test was used. Crude and adjusted ORs with 95\% confidence intervals (CIs) of exposure for enteric infection cases compared with control cases were estimated using conditional logistic regression. All analyses were performed using SAS statistical software (version 9.4 for Windows; SAS Institute, Inc., Cary, NC, USA).

\section{Results}

In total, we identified 17,186 cases of admitted patients with enteric infection and 69,708 applicable control cases. Case demographics are described in Table 1. Within the enteric infection group (case group), gender was represented as $51.9 \%$ male to $48.1 \%$ female. Over $50 \%$ of patients in the case group were older than 50 years of age. Of patients in the case group, $32.84 \%$ were on PPIs, compared to $7.48 \%$ in the control group; $9.9 \%$ of patient in the case group were on H2RAs, compared to $6.9 \%$ in the control group. Of patient in the case group, $8.3 \%$ were on combined PPIs + H2RAs therapy, compared to $2.7 \%$ in the control group. The most common comorbidities along with the case group were hypertension $(54.9 \%)$ and diabetes mellitus (37.0\%). The in-hospital all-cause mortality within the case group was $14.1 \%$.

Table 1. Descriptive analysis of population statistics.

\begin{tabular}{|c|c|c|c|c|c|}
\hline \multirow{2}{*}{ Variable } & \multicolumn{2}{|c|}{ Cases $(N=17,186)$} & \multicolumn{2}{|c|}{ Controls $^{\text {a }}(N=69,708)$} & \multirow{2}{*}{$p$ Value } \\
\hline & N/Percent & Mean \pm SD & N/Percent & Mean \pm SD & \\
\hline Sex & & & & & $0.7479^{a}$ \\
\hline Male & $8920(51.9 \%)$ & - & 36,085 (51.77\%) & - & \\
\hline Female & $8266(48.1 \%)$ & - & $33,623(48.23 \%)$ & - & \\
\hline Age, y & - & $66.68 \pm 17.63$ & - & $66.42 \pm 17.58$ & $0.9810^{a}$ \\
\hline $18-35$ & $1196(6.96 \%)$ & - & $4787(6.87 \%)$ & - & \\
\hline $36-50$ & $1837(10.69 \%)$ & - & $7487(10.74 \%)$ & - & \\
\hline $51-65$ & $3936(22.9 \%)$ & - & $16,080(23.07 \%)$ & - & \\
\hline $65-80$ & $5736(33.38 \%)$ & - & $23,244(33.34 \%)$ & - & \\
\hline$>81$ & $4481(26.07 \%)$ & - & $18,110(25.98 \%)$ & - & \\
\hline PPI Medicine Type & $5644(32.84 \%)$ & - & $5214(7.48 \%)$ & - & $<0.0001$ \\
\hline Pantoprazole & $1195(21.17 \%)$ & - & $1019(19.54 \%)$ & - & \\
\hline Esomeprazole & $3265(57.85 \%)$ & - & $2631(50.46 \%)$ & - & \\
\hline Lansoprazole & $1104(19.56 \%)$ & - & $1406(26.97 \%)$ & - & \\
\hline Dexlansoprazole & $60(1.06 \%)$ & - & $114(2.19 \%)$ & - & \\
\hline Rabeprazole & $20(0.35 \%)$ & - & $20(0.35 \%)$ & - & \\
\hline H2RAs Medicine Type & $1709(9.94 \%)$ & - & $4836(6.94 \%)$ & - & $<0.0001$ \\
\hline Famotidine & $831(48.62 \%)$ & - & $1996(41.27 \%)$ & - & \\
\hline Cimetidine & $878(51.38 \%)$ & - & $2840(58.73 \%)$ & - & \\
\hline PPI or H2RAs Medicine Type & $1424(8.29 \%)$ & & $1907(2.74 \%)$ & & $<0.0001$ \\
\hline Pantoprazole + (Famotidine or Cimetidine) & $264(1.54 \%)$ & - & $304(0.44 \%)$ & - & \\
\hline Esomeprazole + (Famotidine or Cimetidine) & $790(4.6 \%)$ & - & $950(1.36 \%)$ & - & \\
\hline Lansoprazole + (Famotidine or Cimetidine) & $315(1.83 \%)$ & - & $540(0.77 \%)$ & - & \\
\hline Dexlansoprazole + (Famotidine or Cimetidine) & $40(0.23 \%)$ & - & $83(0.12 \%)$ & - & \\
\hline Rabeprazole + (Famotidine or Cimetidine) & $15(0.09 \%)$ & - & $30(0.04 \%)$ & - & \\
\hline
\end{tabular}


Table 1. Cont.

\begin{tabular}{|c|c|c|c|c|c|}
\hline \multirow{2}{*}{ Variable } & \multicolumn{2}{|c|}{ Cases $(N=17,186)$} & \multicolumn{2}{|c|}{ Controls $^{a}(N=69,708)$} & \multirow{2}{*}{$p$ Value } \\
\hline & N/Percent & Mean \pm SD & N/Percent & Mean \pm SD & \\
\hline \multicolumn{5}{|c|}{ Antibiotic use within past $30 \mathrm{~d}$} & $<0.0001$ \\
\hline Yes & $5887(32.73 \%)$ & - & $31,460(43.73 \%)$ & - & \\
\hline No & $12,098(67.27 \%)$ & - & $40,480(56.27 \%)$ & - & \\
\hline \multicolumn{6}{|c|}{ History of } \\
\hline Hypertension & $9428(54.86 \%)$ & - & $41,378(59.36 \%)$ & - & $<0.0001$ \\
\hline Diabetes mellitus & $6353(36.97 \%)$ & - & $29,010(41.62 \%)$ & - & $<0.0001$ \\
\hline Iron deficiency anemia & $2067(12.03 \%)$ & - & $4916(7.05 \%)$ & - & $<0.0001$ \\
\hline Ischemic heart disease & $1866(10.86 \%)$ & - & $5978(8.58 \%)$ & - & $<0.0001$ \\
\hline Acute myocardial infarction & $836(4.86 \%)$ & - & $1339(1.92 \%)$ & - & $<0.0001$ \\
\hline Stroke & $2149(12.5 \%)$ & - & $5877(8.43 \%)$ & - & $<0.0001$ \\
\hline Liver cirrhosis/hepatitis & $2902(16.89 \%)$ & - & $7610(10.92 \%)$ & - & $<0.0001$ \\
\hline Renal failure & $5519(32.11 \%)$ & - & $17,447(25.03 \%)$ & - & $<0.0001$ \\
\hline Gastroesophageal reflux & $1839(10.7 \%)$ & - & $5596(8.03 \%)$ & - & $<0.0001$ \\
\hline Gastric ulcer & $1670(9.72 \%)$ & - & $4151(5.95 \%)$ & - & $<0.0001$ \\
\hline Duodenal ulcer & $1060(6.17 \%)$ & - & $2770(3.97 \%)$ & - & $<0.0001$ \\
\hline Peptic ulcer & $3016(17.55 \%)$ & - & $8537(12.25 \%)$ & - & $<0.0001$ \\
\hline Inflammatory bowel disease & $200(1.16 \%)$ & - & $92(0.13 \%)$ & - & $<0.0001$ \\
\hline Irritable bowel syndrome & $904(5.26 \%)$ & - & $2827(4.06 \%)$ & - & $<0.0001$ \\
\hline Chronic obstruction pulmonary disease & $2009(11.69 \%)$ & - & $6315(9.06 \%)$ & - & $<0.0001$ \\
\hline In hospitai all-cause mortality & $2494(14.08 \%)$ & - & $2694(3.86 \%)$ & - & - \\
\hline
\end{tabular}

Matched by age, sex, and index year. ${ }^{a}$ Chi-square test.

Table 2 shows the association between exposure to PPIs or H2RAs and risk of enteric infection. The adjusted OR for PPIs use with enteric infection was 5.526 (95\% CI, 5.274-5.791). For H2RAs, the adjusted odds ratio was 1.339 (95\% CI, 1.261-1.424). A significant dose response was observed in PPIs or H2RAs use and risk of enteric infection. Subgroups with an OR above average were esomeprazole (OR, 6.319; 95\% CI, 5.959 to 6.7) and pantoprazole (OR, 5.799; 95\% CI, 5.297 to 6.349).

Table 2. Association between exposure to proton pump inhibitors (PPIs), H2-receptor antagonists (H2RAs), or antibiotic use and intestinal infection.

\begin{tabular}{|c|c|c|c|c|}
\hline \multirow[t]{2}{*}{ Exposure } & $\begin{array}{c}\text { Cases } \\
(\mathrm{N}=\mathbf{1 7 , 1 8 6 )}\end{array}$ & $\begin{array}{l}\text { Controls }^{a} \\
(\mathrm{~N}=69,708)\end{array}$ & Crude OR & Adjusted $\mathrm{OR}^{\mathrm{b}}$ \\
\hline & N/Percent & N/Percent & OR $(95 \%$ CI $)$ & OR $(95 \%$ CI $)$ \\
\hline $\begin{array}{c}\text { Among users of PPIs alone } \\
\left(\begin{array}{c}(n=10,858) \\
\text { Drug }\end{array}\right.\end{array}$ & $5644(32.84 \%)$ & $5214(7.48 \%)$ & $6.243(5.98$ to 6.518$) * * *$ & $5.526(5.274$ to 5.791$) * * *$ \\
\hline Pantoprazole & $1195(6.95 \%)$ & $1019(1.46 \%)$ & $6.797(6.232 \text { to } 7.413)^{* * *}$ & $5.799(5.297 \text { to } 6.349)^{* * *}$ \\
\hline Esomeprazole & $3265(19 \%)$ & $2631(3.77 \%)$ & $7.133(6.748 \text { to } 7.540)^{* * *}$ & $6.319(5.959 \text { to } 6.700)^{* * *}$ \\
\hline Lansoprazole & $1104(6.42 \%)$ & $1406(2.02 \%)$ & $4.552(4.195 \text { to } 4.940)^{* * *}$ & $4.043(3.709 \text { to } 4.407)^{* * *}$ \\
\hline Dexlansoprazole & $60(0.35 \%)$ & $114(0.16 \%)$ & 2.985 (2.18 to 4.088$) * * *$ & $2.863(2.070 \text { to } 3.960)^{* * *}$ \\
\hline $\begin{array}{l}\text { Rabeprazole } \\
\text { Daily done }\end{array}$ & $20(0.12 \%)$ & $44(0.06 \%)$ & 2.530 (1.489 to 4.298$)^{* *}$ & $2.112(1.220 \text { to } 3.657)^{* *}$ \\
\hline $\mathrm{cDDD}<=90$ & $2564(14.92 \%)$ & $2749(3.94 \%)$ & $5.362(5.061 \text { to } 5.680)^{* * *}$ & $4.928(4.639 \text { to } 5.236)^{* * *}$ \\
\hline cDDD 91-180 & $1647(9.58 \%)$ & $1498(2.15 \%)$ & $6.388(5.935 \text { to } 6.876)^{* * *}$ & $5.637(5.217 \text { to } 6.091)^{* * *}$ \\
\hline cDDD > 180 & $1433(8.34 \%)$ & $967(1.39 \%)$ & $8.545(7.853 \text { to } 9.298)^{* * *}$ & $7.153(6.541 \text { to } 7.821)^{* * *}$ \\
\hline $\begin{array}{l}\text { Among users of H2RAs alone } \\
\qquad \begin{array}{c}(n=6545) \\
\text { Drug }\end{array}\end{array}$ & $1709(9.94 \%)$ & $4836(6.94 \%)$ & $1.501(1.416$ to 1.592$) * * *$ & $1.339(1.26$ to 1.424$) * * *$ \\
\hline Famotidine & $831(4.84 \%)$ & $2840(4.07 \%)$ & $1.800(1.654 \text { to } 1.959)^{* * *}$ & $1.600(1.466$ to 1.747$) * *$ \\
\hline $\begin{array}{l}\text { Cimetidine } \\
\text { Daily done }\end{array}$ & $878(5.11 \%)$ & $1996(2.86 \%)$ & $1.303(1.205 \text { to } 1.409)^{* * *}$ & $1.164(1.074 \text { to } 1.262)^{* * *}$ \\
\hline $\mathrm{cDDD}<=90$ & $1376(8.01 \%)$ & $3997(5.73 \%)$ & $1.462(1.370 \text { to } 1.559)^{* * *}$ & $1.317(1.232 \text { to } 1.408)^{* * *}$ \\
\hline cDDD 91-180 & $242(1.41 \%)$ & $664(0.95 \%)$ & $1.565(1.348 \text { to } 1.816)^{* * *}$ & $1.366(1.171 \text { to } 1.593)^{* * *}$ \\
\hline
\end{tabular}


Table 2. Cont.

\begin{tabular}{|c|c|c|c|c|}
\hline \multirow{2}{*}{ Exposure } & $\begin{array}{c}\text { Cases } \\
(\mathrm{N}=17,186)\end{array}$ & $\begin{array}{l}\text { Controls }^{a} \\
(\mathrm{~N}=69,708)\end{array}$ & Crude OR & Adjusted OR ${ }^{b}$ \\
\hline & N/Percent & N/Percent & OR (95\% CI) & OR (95\% CI) \\
\hline cDDD $>180$ & $91(0.53 \%)$ & $175(0.25 \%)$ & $2.202(1.704 \text { to } 2.847)^{* * *}$ & $1.727(1.323$ to 2.255$) * * *$ \\
\hline $\begin{array}{l}\text { Among users of PPIs } \\
\text { orH2RAs }(n=3331) \\
\text { Drug }\end{array}$ & $1424(8.29 \%)$ & $1907(2.74 \%)$ & $3.278(3.052$ to 3.520$) * * *$ & $2.416(2.236$ to 2.61$) * * *$ \\
\hline $\begin{array}{c}\text { Pantoprazole }+ \\
\text { (Famotidine or Cimetidine) }\end{array}$ & $264(1.54 \%)$ & $304(0.44 \%)$ & $3.761(3.185 \text { to } 4.440)^{* * *}$ & $2.712(2.280$ to 3.227$) * * *$ \\
\hline $\begin{array}{l}\text { Esomeprazole }+ \\
\text { (Famotidine or Cimetidine) }\end{array}$ & $790(4.6 \%)$ & $950(1.36 \%)$ & 3.655 (3.319 to 4.026$)^{* * *}$ & $2.728(2.464$ to 3.021$) * * *$ \\
\hline $\begin{array}{l}\text { Lansoprazole }+ \\
\text { (Famotidine or Cimetidine) }\end{array}$ & $315(1.83 \%)$ & $540(0.77 \%)$ & $2.563(2.227 \text { to } 2.950)^{* * *}$ & $1.806(1.557 \text { to } 2.096)^{* * *}$ \\
\hline $\begin{array}{c}\text { Dexlansoprazole }+ \\
\text { (Famotidine or Cimetidine) }\end{array}$ & $40(0.23 \%)$ & $83(0.12 \%)$ & $2.149(1.472 \text { to } 3.137)^{* * *}$ & $1.831(1.234$ to 2.716$) *$ \\
\hline $\begin{array}{c}\text { Rabeprazole }+ \\
\text { (Famotidine or Cimetidine) }\end{array}$ & $15(0.09 \%)$ & $30(0.04 \%)$ & $2.231(1.199$ to 4.151$) * *$ & $1.484(0.772$ to 2.853$)$ \\
\hline \multicolumn{5}{|l|}{ Daily done } \\
\hline $\mathrm{cDDD}<=90$ & $846(4.92 \%)$ & $1285(1.84 \%)$ & $2.889(2.643$ to 3.157$) * * *$ & $2.188(1.991$ to 2.405$) * * *$ \\
\hline cDDD 91-180 & $338(1.97 \%)$ & $419(0.60 \%)$ & $3.553(3.073$ to 4.108$) * * *$ & $2.577(2.213$ to 3.000$) * * *$ \\
\hline cDDD > 180 & $240(1.4 \%)$ & $203(0.29 \%)$ & $5.242(4.341$ to 6.329$) * * *$ & $3.561(2.92$ to 4.342$) * * *$ \\
\hline \multicolumn{5}{|l|}{ Antibiotic use within past $30 \mathrm{~d}$} \\
\hline Yes & $5653(32.89 \%)$ & $30464(43.7 \%)$ & $0.627(0.605 \text { to } 0.650)^{* * *}$ & $0.623(0.600$ to 0.646$) * * *$ \\
\hline No & $11533(67.11 \%)$ & $39244(56.3 \%)$ & reference & reference \\
\hline
\end{tabular}

${ }^{a}$ Matched by age, sex, and index date. ${ }^{b}$ Adjusted for by age, sex, and index date, disease history (hypertension, diabetes mellitus iron deficiency anemia, ischemic heart disease, acute myocardial infarction, stroke, liver cirrhosis/hepatitis, renal failure, gastroesophageal reflux, gastric ulcer, duodenal ulcer, peptic ulcer, inflammatory bowel disease, irritable bowel syndrome, chronic obstruction pulmonary disease, gastrointestinal cancer history). ${ }^{*} p<0.05,{ }^{* *} p<0.01,{ }^{* * *} p<0.001$.

Table 3 shows the stratum-specific odds ratios (ORs) for the association between use of PPIs therapy and enteric infection for various subgroups of patients. Subgroups with an OR above average were female (OR, 5.565; 95\% CI, 5.193-5.964), age between 18-65 year-old (OR 6.252-8.565) and patients with a diagnosis of diabetes mellitus (OR, 5.724, 95\% CI, 5.328-6.15).

Table 3. Stratum-specific odds ratios (ORs) for the association between use of proton pump inhibitor therapy and intestinal infection.

\begin{tabular}{|c|c|c|c|c|}
\hline \multirow[b]{2}{*}{ Stratum } & Cases & Controls $^{a}$ & Crude OR & Adjusted OR ${ }^{b}$ \\
\hline & $\begin{array}{c}\text { Exposed/ } \\
\text { Unexposed }\end{array}$ & $\begin{array}{c}\text { Exposed/ } \\
\text { Unexposed }\end{array}$ & OR (95\% CI) & $\operatorname{AOR}(95 \% \mathrm{CI})$ \\
\hline \multicolumn{5}{|l|}{ Sex } \\
\hline Male & $3091 / 5829$ & $2948 / 33,137$ & $6.118(5.772 \text { to } 6.485)^{* * *}$ & $5.476(5.139$ to 5.836$) * * *$ \\
\hline Female & $2553 / 5713$ & $2266 / 31,357$ & $6.398(6.001 \text { to } 6.822)^{* * *}$ & $5.565(5.193$ to 5.964$) * * *$ \\
\hline \multicolumn{5}{|l|}{ Age, y } \\
\hline $18-35$ & $161 / 1035$ & $86 / 4701$ & $8.601(6.556$ to 11.284$) * * *$ & $8.565(6.323 \text { to } 11.602)^{* * *}$ \\
\hline $36-50$ & $476 / 1361$ & $308 / 7179$ & $8.290(7.094 \text { to } 9.686)^{* * *}$ & $7.085(5.957 \text { to } 8.426)^{* * *}$ \\
\hline $51-65$ & $1308 / 2628$ & $1002 / 15,078$ & $7.563(6.894 \text { to } 8.296)^{* * *}$ & $6.252(5.650$ to 6.919$) * * *$ \\
\hline $65-80$ & $2080 / 3656$ & $2094 / 21,150$ & $5.778(5.386$ to 6.199$) * * *$ & $5.264(4.876$ to 5.683$) * * *$ \\
\hline$>81$ & $1619 / 2862$ & $1724 / 16,386$ & $5.396(4.987$ to 5.838$) * * *$ & $4.87(4.474$ to 5.301$) * * *$ \\
\hline \multicolumn{5}{|l|}{ BMI, $\mathrm{kg} / \mathrm{m}^{2}$} \\
\hline$<24$ & $1433 / 2816$ & $1441 / 12,249$ & $4.424(4.063 \text { to } 4.817)^{* * *}$ & $3.923(3.581$ to 4.297$) * * *$ \\
\hline $24-30$ & $1019 / 2078$ & $1200 / 11,869$ & $4.988(4.527 \text { to } 5.497)^{* * *}$ & $4.386(3.949$ to 4.872$) * * *$ \\
\hline$>30$ & $218 / 491$ & $274 / 3333$ & $5.427(4.407 \text { to } 6.682)^{* * *}$ & $4.484(3.564$ to 5.641$) * * *$ \\
\hline \multicolumn{5}{|l|}{ History of } \\
\hline Hypertension & $3339 / 6089$ & $3753 / 37,625$ & $5.542(5.249 \text { to } 5.851)^{* * *}$ & $4.841(4.566$ to 5.133$) * * *$ \\
\hline Diabetes mellitus & $2397 / 3956$ & $2400 / 26,610$ & $6.695(6.266 \text { to } 7.153)^{* * *}$ & $5.724(5.328 \text { to } 6.150)^{* * *}$ \\
\hline Iron deficiency anemia & $817 / 1250$ & $747 / 4169$ & $3.737(3.312$ to 4.218$) * * *$ & $3.646(3.202$ to 4.151$) * * *$ \\
\hline Ischemic heart disease & $774 / 1092$ & $850 / 5128$ & $4.309(3.828 \text { to } 4.851)^{* * *}$ & $3.917(3.452$ to 4.444$) * * *$ \\
\hline
\end{tabular}


Table 3. Cont.

\begin{tabular}{ccccc}
\hline Stratum & Cases & Controls $^{\mathbf{a}}$ & Crude OR & Adjusted OR $^{\mathbf{b}}$ \\
\cline { 2 - 5 } & $\begin{array}{c}\text { Exposed/ } \\
\text { Unexposed }\end{array}$ & $\begin{array}{c}\text { Exposed/ } \\
\text { Unexposed }\end{array}$ & OR (95\% CI) & AOR (95\% CI) \\
\hline Acute myocardial infarction & $408 / 428$ & $292 / 1047$ & $3.467(2.857 \text { to } 4.206)^{* * *}$ & $3.640(2.970 \text { to } 4.461)^{* * *}$ \\
Stroke & $792 / 1357$ & $767 / 5110$ & $3.958(3.518 \text { to } 4.452)^{* * *}$ & $3.760(3.315 \text { to } 4.265)^{* * * *}$ \\
Liver cirrhosis/hepatitis & $1173 / 1729$ & $1128 / 6482$ & $3.897(3.531 \text { to } 4.300)^{* * *}$ & $3.632(3.268 \text { to } 4.038)^{* * *}$ \\
Renal failure & $2280 / 3239$ & $1894 / 15,553$ & $5.916(5.501 \text { to } 6.361)^{* * *}$ & $5.517(5.107 \text { to } 5.96)^{* * *}$ \\
Gastroesophageal reflux & $849 / 990$ & $1424 / 4172$ & $2.612(2.335 \text { to } 2.922)^{* * *}$ & $2.490(2.216 \text { to } 2.797)^{* * * *}$ \\
Gastric ulcer & $826 / 844$ & $1044 / 3107$ & $3.035(2.686 \text { to } 3.429)^{* * *}$ & $2.960(2.602 \text { to } 3.366)^{* * *}$ \\
Duodenal ulcer & $546 / 514$ & $744 / 2026$ & $2.979(2.562 \text { to } 3.463)^{* * *}$ & $2.909(2.484 \text { to } 3.408)^{* * * *}$ \\
Peptic ulcer & $1354 / 1662$ & $2206 / 6331$ & $2.356(2.158 \text { to } 2.572)^{* * *}$ & $2.208(2.014 \text { to } 2.420)^{* * *}$ \\
Inflammatory bowel disease & $39 / 161$ & $14 / 78$ & $1.115(0.481$ to 2.587$)$ & $1.387(0.501$ to 3.839$)$ \\
Irritable bowel syndrome & $304 / 600$ & $482 / 2345$ & $2.596(2.180 \text { to 3.092) })^{* * *}$ & $2.501(2.072 \text { to } 3.019)^{* * *}$ \\
Chronic obstruction & $802 / 1207$ & $862 / 5453$ & $4.227(3.765 \text { to } 4.747)^{* * *}$ & $3.960(3.499 \text { to } 4.482)^{* * *}$ \\
Pulmonary disease & & &
\end{tabular}

${ }^{a}$ Matched by age, sex, and index date. ${ }^{b}$ Adjusted for by age, sex, and index date, disease history (hypertension, diabetes mellitus, iron deficiency anemia, ischemic heart disease, acute myocardial infarction, stroke, liver cirrhosis/hepatitis, renal failure, gastroesophageal reflux, gastric ulcer, duodenitis ulcer, peptic ulcer, inflammatory bowel disease, irritable bowel syndrome, chronic obstruction pulmonary disease, gastrointestinal cancer history) ${ }^{* * *} p<0.001$.

Table 4 shows the stratum-specific ORs for the association between use of H2RAs therapy and enteric infection for various subgroups of patients. For subgroup of ischemic heart disease, acute myocardial infarction, liver cirrhosis/hepatitis, gastroesophageal reflux, gastric ulcer, duodenal ulcer and irritable bowel syndrome, there is no obvious association between use of H2RAs therapy and intestinal infection ( OR less than 1)

Table 4. Stratum-specific odds ratios (ORs) for the association between use of H2-receptor antagonists therapy and intestinal infection.

\begin{tabular}{|c|c|c|c|c|}
\hline \multirow[b]{2}{*}{ Stratum } & Cases & Controls ${ }^{a}$ & Crude OR & Adjusted $\mathrm{OR}^{\mathrm{b}}$ \\
\hline & $\begin{array}{c}\text { Exposed/ } \\
\text { Unexposed }\end{array}$ & $\begin{array}{c}\text { Exposed/ } \\
\text { Unexposed }\end{array}$ & OR (95\% CI) & AOR $(95 \%$ CI) \\
\hline \multicolumn{5}{|l|}{ Sex } \\
\hline Male & $842 / 8078$ & $2332 / 33,753$ & $1.526(1.404 \text { to } 1.659)^{* * *}$ & $1.349(1.236 \text { to } 1.471)^{* * *}$ \\
\hline Female & $867 / 7399$ & $2504 / 31,119$ & $1.478(1.361 \text { to } 1.605)^{* * * *}$ & $1.328(1.219 \text { to } 1.448)^{* * *}$ \\
\hline \multicolumn{5}{|l|}{ Age, y } \\
\hline $18-35$ & $64 / 1132$ & $84 / 4703$ & $3.191(2.287$ to 4.452$) * * *$ & $2.939(2.062 \text { to } 4.191)^{* * *}$ \\
\hline $36-50$ & $163 / 1674$ & $232 / 7255$ & $3.074(2.497 \text { to } 3.786)^{* * *}$ & $2.563(2.053 \text { to } 3.201)^{* * *}$ \\
\hline $51-65$ & $400 / 3536$ & $972 / 15,108$ & 1.772 (1.568 to 2.004$)^{* * *}$ & $1.557(1.369 \text { to } 1.771)^{* * *}$ \\
\hline $65-80$ & $590 / 5146$ & $1892 / 21,352$ & $1.305(1.183 \text { to } 1.44)^{* * *}$ & $1.194(1.078$ to 1.322$) * *$ \\
\hline$>81$ & $492 / 3989$ & $1656 / 16,454$ & $1.238(1.112 \text { to } 1.379)^{* * *}$ & $1.117(0.998$ to 1.250$)$ \\
\hline \multicolumn{5}{|l|}{ BMI, $\mathrm{kg} / \mathrm{m}^{2}$} \\
\hline$<24$ & $556 / 3693$ & $1471 / 12,219$ & $1.285(1.156 \text { to } 1.428)^{* * *}$ & $1.201(1.076 \text { to } 1.339)^{* * *}$ \\
\hline $24-30$ & $448 / 2649$ & $1441 / 11,628$ & $1.367(1.218 \text { to } 1.534)^{* * *}$ & $1.289(1.144 \text { to } 1.452)^{* * *}$ \\
\hline$>30$ & $92 / 617$ & $380 / 3227$ & 1.188 (0.926 to 1.524$)$ & $1.148(0.886$ to 1.489$)$ \\
\hline \multicolumn{5}{|l|}{ History of } \\
\hline Hypertension & $1093 / 8335$ & $3623 / 37,755$ & $1.347(1.252 \text { to } 1.448)^{* * *}$ & $1.199(1.112 \text { to } 1.292)^{* * *}$ \\
\hline Diabetes mellitus & $684 / 5669$ & $2192 / 26,818$ & 1.425 (1.300 to 1.562$)^{* * * *}$ & $1.237(1.125 \text { to } 1.361)^{* * *}$ \\
\hline Iron deficiency anemia & $212 / 1855$ & $416 / 4500$ & $1.157(0.969 \text { to } 1.381)^{* * *}$ & $1.074(0.895$ to 1.290$)$ \\
\hline Ischemic heart disease & $226 / 1640$ & $889 / 5089$ & $0.773(0.658 \text { to } 0.906)^{* *}$ & $0.801(0.680 \text { to } 0.943)^{* *}$ \\
\hline Acute myocardial infarction & $81 / 755$ & $171 / 1168$ & $0.695(0.521 \text { to } 0.926)^{*}$ & $0.714(0.532$ to 0.960$) *$ \\
\hline Stroke & $289 / 1860$ & $722 / 5155$ & $1.093(0.942$ to 1.269$)$ & $1.084(0.929$ to 1.265$)$ \\
\hline Liver cirrhosis/hepatitis & $326 / 2576$ & $924 / 6686$ & $0.935(0.816$ to 1.072$)$ & $0.936(0.813$ to 1.078$)$ \\
\hline Renal failure & $582 / 4937$ & $1524 / 15,923$ & $1.194(1.078$ to 1.322$) * *$ & $1.104(0.994$ to 1.226$)$ \\
\hline Gastroesophageal reflux & $175 / 1664$ & $764 / 4832$ & $0.660(0.553 \text { to } 0.787)^{* * * *}$ & $0.664(0.554 \text { to } 0.795)^{* * *}$ \\
\hline Gastric ulcer & $132 / 1538$ & $378 / 3773$ & 0.847 (0.687 to 1.046$)$ & $0.819(0.660$ to 1.016$)$ \\
\hline Duodenal ulcer & $66 / 994$ & $196 / 2574$ & $0.856(0.637$ to 1.151$)$ & $0.792(0.585$ to 1.071$)$ \\
\hline Peptic ulcer & $361 / 2655$ & $1379 / 7158$ & $0.722(0.637 \text { to } 0.819)^{* * *}$ & $0.746(0.656 \text { to } 0.848)^{* * *}$ \\
\hline
\end{tabular}


Table 4. Cont.

\begin{tabular}{ccccc}
\hline \multirow{2}{*}{ Stratum } & Cases & Controls ${ }^{\mathbf{a}}$ & Crude OR & Adjusted OR $^{\mathbf{b}}$ \\
\cline { 2 - 5 } & $\begin{array}{c}\text { Exposed/ } \\
\text { Unexposed }\end{array}$ & $\begin{array}{c}\text { Exposed/ } \\
\text { Unexposed }\end{array}$ & OR (95\% CI) & AOR (95\% CI) \\
\hline $\begin{array}{c}\text { Inflammatory bowel disease } \\
\text { Irritable bowel syndrome }\end{array}$ & $18 / 182$ & $8 / 84$ & $0.921(0.321$ to 2.643) & $1.018(0.318$ to 3.259$)$ \\
$\begin{array}{c}\text { Chronic obstruction } \\
\text { pulmonary disease }\end{array}$ & $132 / 772$ & $454 / 2373$ & $0.927(0.747$ to 1.149$)$ & $0.942(0.753$ to 1.177$)$ \\
\hline
\end{tabular}

${ }^{a}$ Matched by age, sex, and index date. ${ }^{b}$ Adjusted for by age, sex, and index date, disease history (hypertension, diabetes mellitus, iron deficiency anemia, ischemic heart disease, acute myocardial infarction, stroke, liver cirrhosis/hepatitis, renal failure, gastroesophageal reflux, gastric ulcer, duodenal ulcer, peptic ulcer, inflammatory bowel disease, irritable bowel syndrome, chronic obstruction pulmonary disease, gastrointestinal cancer history) ${ }^{*} p<0.05,{ }^{* *} p<0.01,{ }^{* * *} p<0.001$.

The most common etiological pathogens were Enterococcus (44.8\%), Clostridioides difficile (34.5\%), and Salmonella spp. (10.2\%), as shown in Table 5.

Table 5. Isolated microorganisms of the intestinal infection by the stool examination of causative bacteria.

\begin{tabular}{ccc}
\hline Causative Bacteria & $\begin{array}{c}\text { Intestinal Infection } \\
(\mathbf{N}=\mathbf{8 9 2 3})\end{array}$ & Percentage \\
\hline Acineto & 2 & $0.02 \%$ \\
Aerobes & 11 & $0.12 \%$ \\
Aeromonas & 36 & $0.40 \%$ \\
Campy and Campylobacter & 263 & $2.95 \%$ \\
Candida & 45 & $0.50 \%$ \\
Clostridioides difficile & 3076 & $34.47 \%$ \\
Enterococcus & 3995 & $44.77 \%$ \\
E.coli & 3 & $0.03 \%$ \\
Klebsiella & 10 & $0.11 \%$ \\
Plesiomonas shigelloides & 33 & $0.37 \%$ \\
Ps. and Pseudomonas sp. & 421 & $4.72 \%$ \\
Sal. and Salmonella sp. & 906 & $10.15 \%$ \\
Shigella & 14 & $0.16 \%$ \\
Staph & 22 & $0.25 \%$ \\
Vibrio & 28 & $0.31 \%$ \\
Yeast-like & 58 & $0.65 \%$ \\
\hline
\end{tabular}

\section{Discussions}

To our knowledge, this is the first large-scale cohort study investigating the association between gastric-acid-suppressive drug use and the occurrence of enteric infection for Asia population. A significant dose response was also observed The current study suggests that use of gastric-acid-suppressive drugs increases the risk of enteric infection. The adjusted odds ratio (OR) between PPI use and enteric infection was 5.526 (95\% confidence interval [CI], 5.274-5.791). For H2RAs, the adjusted odds ratio was 1.339 (95\% confidence interval [CI], 1.261-1.424).

Gastric acid is bactericidal and is an important defense mechanism against ingested microorganisms. PPIs, which decrease the secretion of hydrochloric acid, may inadvertently make the stomach a more hospitable environment to ingested pathogens. Gastric acid has a $\mathrm{pH}<4$ and is known to eliminate exogenous acid sensitive bacteria within $15 \mathrm{~min}$. A recent study by sequenced $16 \mathrm{~S}$ rRNA from a fecal sample revealed that oral microbiome is more abundant in the gut microbiome among those taking PPIs. Moreover, PPI-induced hypochlorhydria facilitates colonization of more distal parts of the digestive tract by upper gastrointestinal microbiota PPI use was associated with increases in the Lactobacillales order, and in particular the family Streptococcaceae [21-23].

A link between PPIs and increased enteric infection risk is based on several potential mechanisms. PPIs can alter the gut microbiota, leading to dysbiosis and impaired gut barrier function that results in compromise to gut immunity and susceptibility to various 
enteric pathogens [21,24]. PPIs negatively influence the function of polymorphonuclear cells, specifically with respect to phagocytosis, oxidative burst, chemotaxis, and cytotoxic activity, weakening the immune system. Gastric acid acts as a barrier to progression down the GI tract for pharyngeal and environmental bacteria. The usage of PPIs also affects the gut microenvironment by modifying $\mathrm{pH}$ in the stomach and small intestine, allowing colonisation by these bacteria further along the GI tract and is proven to cause gut dysbiosis. Moreover, PPIs are associated with a significant decrease in Shannon's diversity index and with changes in $20 \%$ of the bacterial taxa. Multiple oral bacteria tend to be over-represented in the fecal microbiome among PPI users [21].

PPI-induced hypochlorhydria increase the risk of respiratory infection by permitting the transmission of ingested pathogens into the respiratory system. A meta-analysis performed in 2011 found that the risk of community-acquired pneumonia was $34 \%$ higher in patients on PPIs, which increased with higher dosing [25].

Long-term use of PPI is associated with small intestinal bacterial overgrowth (SIBO), likely due to hypochlorhydria and loss of gastric defense [26]. According to another meta-analysis, PPI use is associated with an 8-fold relative increased risk of SIBO [27].

PPI could cause profound changes to the colonic microbiota, decrease in the abundance of commensal bacteria, reduce microbial diversity, and increase oral bacteria in stool $[21,22,28]$. Therefore, PPI-driven dysbiosis increases the risk of enteric infections by Clostridium difficile, Salmonella, Campylobacter, and diarrheagenic Escherichia coli.

Retrospective case-control studies show an approximately 3-fold relative risk for Salmonella or Campylobacter infections after exposure to PPIs [29]. In our study, $10.2 \%$ of causative bacteria were Salmonella spp. Besides diabetes, autoimmune diseases, cirrhosis, and recent antibiotic use, PPIs have also been associated with Salmonella infections [30]. Regarding the possible association between PPI use and bacterial enteric infections, one meta-analysis encompassing over 10,000 patients found a pooled OR of 3.33 [11,15]. A recent meta-analysis showed that use of gastric-acid suppressants may be a risk factor for enteric peritonitis in patients undergoing peritoneal dialysis [4].

PPI use has been linked with increased risk of both incidental and recurrent Clostridioides difficile infection (CDi) [31]. According to a systematic review and meta-analysis study, PPI users have a $74 \%$ higher risk of developing $\mathrm{CDi}$, as well as a 2.5 -fold higher risk of recurrent infections, compared with nonusers [16] Alterations in gut bacteria due to hypochlorhydria may lead to pathogen colonization [21,28]. Seo et al. reports that risk of CDi was significantly greater among groups receiving PPIs and/or H2RAs than among matched controls case (PPIs vs control: HR, 2.65; 95\% CI 1.28-5.79; $p=0.011$; H2RAs vs control: HR 2.43; 95\% CI 1.09-5.68; $p=0.034$ ) [32]. Wariness of enteric infection is, therefore, warranted for patients on gastric-acid-suppressive drugs and cessation of unnecessary gastric acid suppressive drugs should be considered at the time of CDI diagnosis [31].

PPIs are a risk factor for hepatic encephalopathy and spontaneous bacterial peritonitis in patients with cirrhosis [33]. Studies show a 2-fold relative risk for spontaneous bacterial peritonitis associated with exposure to PPIs [34]. These findings are consistent with the hypothesis that PPIs may increase translocation of gut bacteria and then facilitating the spread of pathogens and bacterial metabolism products. Furthermore, recent study revealed that gut dysbiosis plays an important role in hepatic encephalopathy [35,36]. PPIs use in decompensated cirrhosis is associated with increased risk of mortality and hepatic decompensation [37]. Moreover, PPIs exposure with cDDD $>90$ is associated with higher mortality, [aHR $=2.27,(1.10-5.14) ; \mathrm{P}=0.038$, compared to non-users] [38]. In our current study, the adjusted odds ratio (OR) of PPIs use and enteric infection for patient with liver cirrhosis/hepatitis was 3.632 (95\% confidence interval [CI], 3.268-4.038).

For the association between PPIs therapy and enteric infection, subgroups with an OR above average were female (OR, 5.565; 95\% CI, 5.193-5.964), age between 18 65 year-old (OR 6.252 8.565) and patients with a diagnosis of diabetes mellitus (OR, 5.724, 95\% CI, 5.328-6.15). Diabetes mellitus is a risk factor for enteric infection with Salmonella, too [30]. Gastric acid suppressive drugs should be used cautionary in diabetic patient. For subgroup 
of ischemic heart disease, acute myocardial infarction and liver cirrhosis/hepatitis, there is no obvious association between use of H2RAs therapy and enteric infection (OR less than 1). Further prospective investigation is warranted to compare the risk of enteric infection among PPi and H2RAs therapy in such individual.

The strengths of the current study include the large numbers of case patients and controls. The study also had some limitations that need to be considered when interpreting the results. First, it is a retrospective study. PPI or H2RA use was measured using physician prescriptions available in electronic system. Patient compliance with prescribed medication is unknown. Presumes that all medications were taken by the patients as prescribed may overestimate the actual ingested dosage. Patients may not have been identified if their drug was obtained over-the counter. Second, although all results are standardized for age and sex, residual confounding factors are ever present. As acid-suppressive therapy is frequently used in patients with gastrointestinal disorders and in patients prescribed NSAIDs or aspirin, analysis restricted to patients without prior gastrointestinal diseases or prior NSAID exposure was not performed.

In conclusion, our results suggest that acid-suppressive drugs such as PPIs and H2RAs are associated with an increased risk of enteric infection. More potent acid inhibition is potentially associated with an increased risk. Therefore, acid-suppressive drugs should be prescribed with caution and with full consideration of appropriate dosing and duration.

Author Contributions: Conceptualization, C.-J.K. and C.-Y.L.; methodology, C.-Y.H.; software, C.-Y.H.; validation, C.-W.C. and W.-R.L.; formal analysis, C.-Y.H.; investigation, C.-Y.L.; resources, C.-W.C.; data curation, C.-T.C.; writing-original draft preparation, C.-J.K.; writing-review and editing, C.-J.K. and S.-Y.H. visualization, C.-T.C.; supervision, S.-Y.H.; project administration, W.-R.L.; funding acquisition, W.-R.L. All authors have read and agreed to the published version of the manuscript.

Funding: This study was founded by CMRP (CORPG3J0511, CORPG3J0211, CMRPG3K0691, and CMRPG3K1811).

Institutional Review Board Statement: Ethics approval was obtained from the Institutional Review Board of CGMH (No.201900774B0C601). As all data were anonymized from existing databases and results were presented in aggregate.

Informed Consent Statement: The requirement for informed consent was waived according to IRB regulations.

Data Availability Statement: The data presented in this study are available on request from the corresponding author.

Acknowledgments: The authors also express their gratitude in being granted access to CGRD from Chang Gung Memorial Hospital. The authors thank C.-Y.H. and H.-C.H. for their assistance with statistical analysis and wish to acknowledge the support provided by the Maintenance Project of the Center for Big Data Analytics and Statistics (Grant CLRPG3D0048). The content of this study solely represented the views of the authors but not the official view of Chang Gung Memorial Hospital.

Conflicts of Interest: The authors report no conflict of interest.

\section{References}

1. Freedberg, D.E.; Kim, L.S.; Yang, Y.X. The Risks and Benefits of Long-term Use of Proton Pump Inhibitors: Expert Review and Best Practice Advice From the American Gastroenterological Association. Gastroenterology 2017, 152, 706-715. [CrossRef] [PubMed]

2. Fock, K.M.; Talley, N.; Goh, K.L.; Sugano, K.; Katelaris, P.; Holtmann, G.; Pandolfino, J.E.; Sharma, P.; Ang, T.L.; Hongo, M.; et al. Asia-Pacific consensus on the management of gastro-oesophageal reflux disease: An update focusing on refractory reflux disease and Barrett's oesophagus. Gut 2016, 65, 1402-1415. [CrossRef]

3. Katz, P.O.; Gerson, L.B.; Vela, M.F. Guidelines for the diagnosis and management of gastroesophageal reflux disease. Am. J. Gastroenterol. 2013, 108, 308-328. [CrossRef] [PubMed]

4. Zhong, H.J.; Lin, D.; Lu, Z.Y.; Yang, W.Y.; Chen, Y. Use of gastric-acid suppressants may be a risk factor for enteric peritonitis in patients undergoing peritoneal dialysis: A meta-analysis. J. Clin. Pharm. Ther. 2019, 44, 209-215. [CrossRef] [PubMed]

5. Malfertheiner, P.; Kandulski, A.; Venerito, M. Proton-pump inhibitors: Understanding the complications and risks. Nat. Rev. Gastroenterol. Hepatol. 2017, 14, 697-710. [CrossRef] [PubMed]

6. Yang, Y.X.; Metz, D.C. Safety of proton pump inhibitor exposure. Gastroenterology 2010, 139, 1115-1127. [CrossRef] 
7. Wang, Y.F.; Chen, Y.T.; Luo, J.C.; Chen, T.J.; Wu, J.C.; Wang, S.J. Proton-Pump Inhibitor Use and the Risk of First-Time Ischemic Stroke in the General Population: A Nationwide Population-Based Study. Am. J. Gastroenterol. 2017, 112, 1084-1093. [CrossRef]

8. Moayyedi, P.; Leontiadis, G.I. The risks of PPI therapy. Nat. Rev. Gastroenterol. Hepatol. 2012, 9, 132-139. [CrossRef]

9. Eusebi, L.H.; Rabitti, S.; Artesiani, M.L.; Gelli, D.; Montagnani, M.; Zagari, R.M.; Bazzoli, F. Proton pump inhibitors: Risks of long-term use. J. Gastroenterol. Hepatol. 2017, 32, 1295-1302. [CrossRef]

10. Islam, M.M.; Poly, T.N.; Walther, B.A.; Dubey, N.K.; Anggraini Ningrum, D.N.; Shabbir, S.A.; Jack Li, Y.C. Adverse outcomes of long-term use of proton pump inhibitors: A systematic review and meta-analysis. Eur J. Gastroenterol. Hepatol. 2018, 30, 1395-1405. [CrossRef]

11. Vaezi, M.F.; Yang, Y.X.; Howden, C.W. Complications of Proton Pump Inhibitor Therapy. Gastroenterology 2017, 153, 35-48. [CrossRef] [PubMed]

12. Brusselaers, N.; Sadr-Azodi, O.; Engstrand, L. Long-term proton pump inhibitor usage and the association with pancreatic cancer in Sweden. J. Gastroenterol. 2020, 55, 453-461. [CrossRef] [PubMed]

13. Sanduleanu, S.; Jonkers, D.; de Bruïne, A.; Hameeteman, W.; Stockbrügger, R.W. Changes in gastric mucosa and luminal environment during acid-suppressive therapy: A review in depth. Dig. Liver Dis. 2001, 33, 707-719. [CrossRef]

14. Handa, O.; Yoshida, N.; Fujita, N.; Tanaka, Y.; Ueda, M.; Takagi, T.; Kokura, S.; Naito, Y.; Okanoue, T.; Yoshikawa, T. Molecular mechanisms involved in anti-inflammatory effects of proton pump inhibitors. Inflamm. Res. 2006, 55, 476-480. [CrossRef] [PubMed]

15. Leonard, J.; Marshall, J.K.; Moayyedi, P. Systematic review of the risk of enteric infection in patients taking acid suppression. Am. J. Gastroenterol. 2007, 102, 2047-2056, quiz 2057. [CrossRef]

16. Kwok, C.S.; Arthur, A.K.; Anibueze, C.I.; Singh, S.; Cavallazzi, R.; Loke, Y.K. Risk of Clostridium difficile infection with acid suppressing drugs and antibiotics: Meta-analysis. Am. J. Gastroenterol. 2012, 107, 1011-1019. [CrossRef]

17. Dial, S.; Delaney, J.A.; Barkun, A.N.; Suissa, S. Use of gastric acid-suppressive agents and the risk of community-acquired Clostridium difficile-associated disease. Jama 2005, 294, 2989-2995. [CrossRef]

18. Trifan, A.; Stanciu, C.; Girleanu, I.; Stoica, O.C.; Singeap, A.M.; Maxim, R.; Chiriac, S.A.; Ciobica, A.; Boiculese, L. Proton pump inhibitors therapy and risk of Clostridium difficile infection: Systematic review and meta-analysis. World J. Gastroenterol. 2017, 23, 6500-6515. [CrossRef] [PubMed]

19. Goldstein, J.A.; Ishizaki, T.; Chiba, K.; de Morais, S.M.; Bell, D.; Krahn, P.M.; Evans, D.A. Frequencies of the defective CYP2C19 alleles responsible for the mephenytoin poor metabolizer phenotype in various Oriental, Caucasian, Saudi Arabian and American black populations. Pharmacogenetics 1997, 7, 59-64. [CrossRef]

20. Tsai, M.S.; Lin, M.H.; Lee, C.P.; Yang, Y.H.; Chen, W.C.; Chang, G.H.; Tsai, Y.T.; Chen, P.C.; Tsai, Y.H. Chang Gung Research Database: A multi-institutional database consisting of original medical records. Biomed. J. 2017, 40, 263-269. [CrossRef] [PubMed]

21. Imhann, F.; Bonder, M.J.; Vich Vila, A.; Fu, J.; Mujagic, Z.; Vork, L.; Tigchelaar, E.F.; Jankipersadsing, S.A.; Cenit, M.C.; Harmsen, H.J.; et al. Proton pump inhibitors affect the gut microbiome. Gut 2016, 65, 740-748. [CrossRef]

22. Macke, L.; Schulz, C.; Koletzko, L.; Malfertheiner, P. Systematic review: The effects of proton pump inhibitors on the microbiome of the digestive tract-evidence from next-generation sequencing studies. Aliment. Pharmacol. Ther. 2020, 51, 505-526. [CrossRef]

23. Corsonello, A.; Lattanzio, F.; Bustacchini, S.; Garasto, S.; Cozza, A.; Schepisi, R.; Lenci, F.; Luciani, F.; Maggio, M.G.; Ticinesi, A.; et al. Adverse Events of Proton Pump Inhibitors: Potential Mechanisms. Curr. Drug Metab. 2018, 19, 142-154. [CrossRef]

24. DuPont, A.W.; DuPont, H.L. The intestinal microbiota and chronic disorders of the gut. Nat. Rev. Gastroenterol. Hepatol. 2011, 8, 523-531. [CrossRef] [PubMed]

25. Eom, C.S.; Jeon, C.Y.; Lim, J.W.; Cho, E.G.; Park, S.M.; Lee, K.S. Use of acid-suppressive drugs and risk of pneumonia: A systematic review and meta-analysis. CMAJ 2011, 183, 310-319. [CrossRef]

26. Fujimori, S. What are the effects of proton pump inhibitors on the small intestine? World J. Gastroenterol. 2015, 21, 6817-6819. [CrossRef]

27. Lo, W.K.; Chan, W.W. Proton pump inhibitor use and the risk of small intestinal bacterial overgrowth: A meta-analysis. Clin. Gastroenterol. Hepatol. 2013, 11, 483-490. [CrossRef]

28. Bruno, G.; Zaccari, P.; Rocco, G.; Scalese, G.; Panetta, C.; Porowska, B.; Pontone, S.; Severi, C. Proton pump inhibitors and dysbiosis: Current knowledge and aspects to be clarified. World J. Gastroenterol. 2019, 25, 2706-2719. [CrossRef] [PubMed]

29. Bavishi, C.; Dupont, H.L. Systematic review: The use of proton pump inhibitors and increased susceptibility to enteric infection. Aliment. Pharmacol. Ther. 2011, 34, 1269-1281. [CrossRef]

30. Wu, H.H.; Chen, Y.T.; Shih, C.J.; Lee, Y.T.; Kuo, S.C.; Chen, T.L. Association between recent use of proton pump inhibitors and nontyphoid salmonellosis: A nested case-control study. Clin. Infect. Dis 2014, 59, 1554-1558. [CrossRef] [PubMed]

31. McDonald, E.G.; Milligan, J.; Frenette, C.; Lee, T.C. Continuous Proton Pump Inhibitor Therapy and the Associated Risk of Recurrent Clostridium difficile Infection. JAMA Intern. Med. 2015, 175, 784-791. [CrossRef]

32. Seo, S.I.; You, S.C.; Park, C.H.; Kim, T.J.; Ko, Y.S.; Kim, Y.; Yoo, J.J.; Kim, J.; Shin, W.G. Comparative risk of Clostridium difficile infection between proton pump inhibitors and histamine-2 receptor antagonists: A 15-year hospital cohort study using a common data model. J. Gastroenterol. Hepatol. 2020, 35, 1325-1330. [CrossRef] [PubMed]

33. Dam, G.; Vilstrup, H.; Watson, H.; Jepsen, P. Proton pump inhibitors as a risk factor for hepatic encephalopathy and spontaneous bacterial peritonitis in patients with cirrhosis with ascites. Hepatology 2016, 64, 1265-1272. [CrossRef] [PubMed] 
34. Xu, H.B.; Wang, H.D.; Li, C.H.; Ye, S.; Dong, M.S.; Xia, Q.J.; Zhang, A.Q.; Pan, K.; Ge, X.L.; Dong, J.H. Proton pump inhibitor use and risk of spontaneous bacterial peritonitis in cirrhotic patients: A systematic review and meta-analysis. Genet. Mol. Res. 2015, 14, 7490-7501. [CrossRef] [PubMed]

35. Sung, C.M.; Lin, Y.F.; Chen, K.F.; Ke, H.M.; Huang, H.Y.; Gong, Y.N.; Tsai, W.S.; You, J.F.; Lu, M.J.; Cheng, H.T.; et al. Predicting Clinical Outcomes of Cirrhosis Patients With Hepatic Encephalopathy From the Fecal Microbiome. Cell Mol. Gastroenterol. Hepatol. 2019, 8, 301-318.e302. [CrossRef] [PubMed]

36. Zhu, J.; Qi, X.; Yu, H.; Yoshida, E.M.; Mendez-Sanchez, N.; Zhang, X.; Wang, R.; Deng, H.; Li, J.; Han, D.; et al. Association of proton pump inhibitors with the risk of hepatic encephalopathy during hospitalization for liver cirrhosis. United Eur. Gastroenterol. J. 2018, 6, 1179-1187. [CrossRef]

37. Nardelli, S.; Gioia, S.; Ridola, L.; Farcomeni, A.; Merli, M.; Riggio, O. Proton Pump Inhibitors Are Associated With Minimal and Overt Hepatic Encephalopathy and Increased Mortality in Patients With Cirrhosis. Hepatology 2019, 70, 640-649. [CrossRef]

38. De Roza, M.A.; Kai, L.; Kam, J.W.; Chan, Y.H.; Kwek, A.; Ang, T.L.; Hsiang, J.C. Proton pump inhibitor use increases mortality and hepatic decompensation in liver cirrhosis. World J. Gastroenterol. 2019, 25, 4933-4944. [CrossRef] [PubMed] 